\title{
Total Knee Arthroplasty using Computer Assisted Surgery in Paget 's disease of Knee-A case report
}

\author{
Eachempati $\mathrm{KK}^{* 1}$, Dannana $\mathrm{CS}^{2}$ and Boyapati $\mathrm{G}^{3}$ \\ ${ }^{1}$ Chief Consultant and Director Orthopaedics, Max Cure Hospitals, India \\ ${ }^{2}$ Consultant Orthopaedic Surgeon, Max Cure Hospitals, India \\ ${ }^{3}$ Clinical Fellow in Arthroplasty, Max Cure Hospitals, India
}

Received: October 31,2017; Published: November 09, 2017

*Corresponding author: Eachempati KK, Fellow in Computer Navigation, Chief Consultant and Director Orthopaedics, Max Cure Hospitals, Hyderabad, India; Email: kke1975@gmail.com

\begin{abstract}
The purpose of this case report is to highlight the advantages of computer assisted navigation surgery (CAS) to improve the accuracy in performing Total Knee Arthroplasty (TKA) in a patient affected by Paget's disease of the femur and secondary osteoarthrosis of knee. Paget's disease can be associated with technical difficulties while performing knee arthroplasty because of intra and/or extra-articular deformity in either sagittal or coronal planes or both and there are published reports of sub-optimal varus or valgus placement of components by the manual technique. A 55-year-old male with monostotic Paget's involvement of the femur with lateral femoral bowing and end stage arthrosis in the knee was managed with a primary cemented cruciate-retaining TKA using Aesculap Orthopilot 5.0 navigation system. His pre-op VAS score of $8 / 10$ and Oxford Knee Score of 20 improved to $2 / 10$ and 42 respectively at 1 year follow up. Knee involvement with Paget's in Indian population is extremely rare and we are not aware of any previous case reports of using CAS in performing TKA in Paget's. CAS can obviate the need for corrective osteotomy when performing TKA in patients with extra-articular deformity.
\end{abstract}

Abbreviations: TKA: Total Knee Arthroplasty; CAS: Computer Assisted Navigation Surgery; VAS: Visual Analogue Scale; OKS: Oxford Knee Score

\section{Introduction}

Paget's disease is a disorder of bone characterized by increased bone turnover, enlargement and thickening of the bone which is unusually brittle, more prone for deformity or fractures in weight bearing joints and arthritis of joints with affected adjacent bones [1]. As the proximal femur and pelvis are more commonly affected, hip arthritis and challenges with total hip arthroplasty [2,3] are well described than the knee arthritis and total knee arthroplasty [4]. We report a case of monostotic Paget's involvement of distal femur and associated knee arthritis that underwent a total knee arthroplasty by computer assisted surgery. Paget's involvement especially isolated involvement of distal femur and knee in Indian population is rare as compared to the Western population [5]. We are not aware of any previous reports of using computer assisted surgery in Paget's involvement of the knee. The purpose of this report is to highlight the role of computer assisted surgery in achieving optimal component alignment in cases of intra or extra articular deformity which is seen with Paget's disease.

\section{Case Details}

A 56-year-old male presented to us with severe disabling pain in his right knee affecting his day to day activities and which failed to respond to conservative measures. His plain radiographs showed mixed sclerotic and lytic lesions involving the entire shaft of the femur and a diagnosis of Paget's was made on the typical radiological signs [6] on plain radiographs (Figures $1 \& 2$ ) and MRI (Figure 3). There was an anterolateral bowing of the femur about 100 and knee arthrosis changes with intra-articular varus deformity. There was no involvement of the tibia (Figure 2). His pre-operative pain score was 8 on visual analogue scale (VAS) and Oxford Knee Score [7] (OKS) was 20. The patient underwent a primary cemented cruciate retaining TKA by computer assisted navigation system (B Braun Columbus, Aesculap Orthopilot 5.0 navigation). The patient was placed supine, under spinal anaesthetic and tourniquet control a midline incision with sub vastus arthrotomy was performed. Using femur and tibia infra-red trackers, registration of the the 
following bony landmarks was made-posterior most part of medial and lateral femoral condyles, lowest point of medial tibial plateau, highest point of lateral tibial plateau, knee centre, anterior femoral cortex, most prominent points of medial and lateral malleoli and hip, knee and ankle centres were marked.

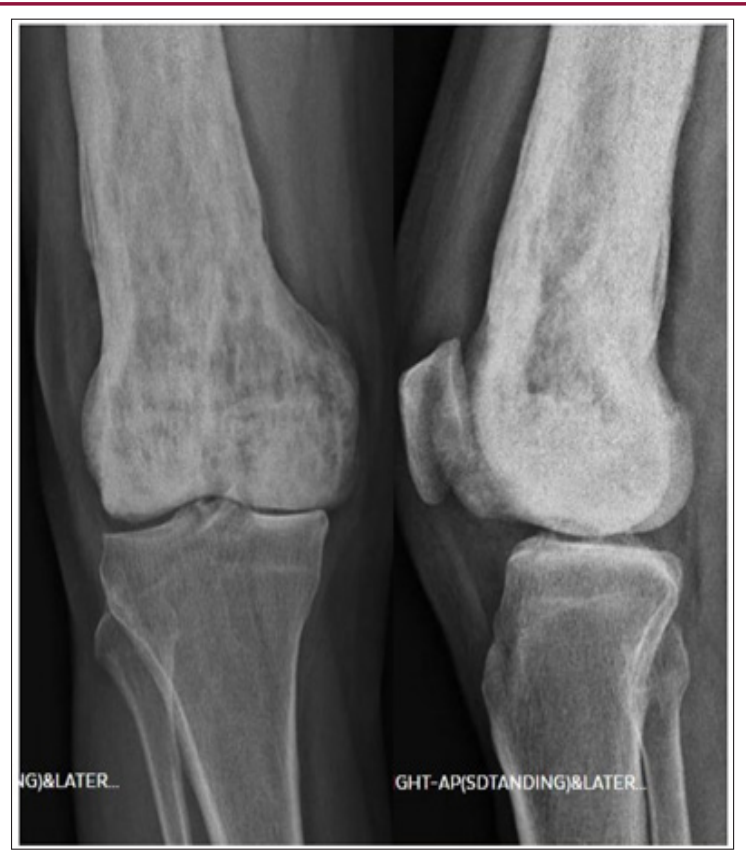

Figure 1: Plain radiograph showing the mixed sclerotic and lytic lesion typical of Paget's involving distal femur with arthritic changes in the knee. Note: There is no tibial side involvement with Paget's.

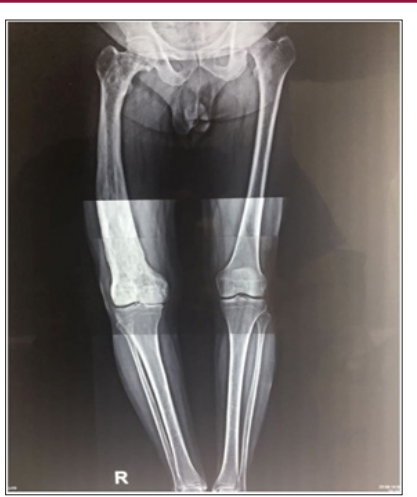

Figure 2: Long leg radiographs showing the characteristic lateral femoral bowing and involvement of the entire femur with Paget's. Notice the normal pelvis and contralateral femur.

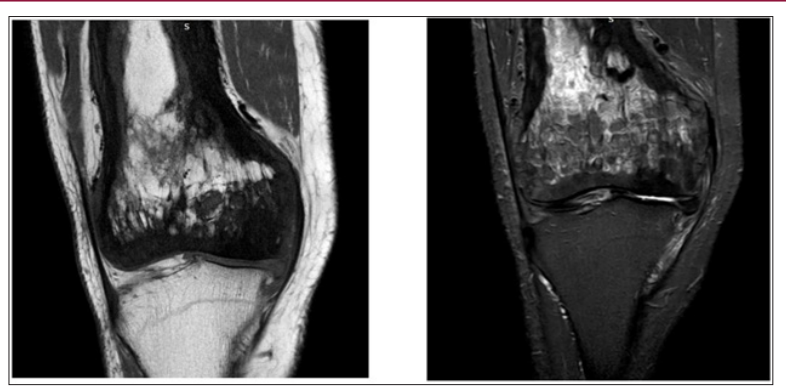

Figure 3: The typical features of Paget's on T2 and T1 weighted MRI images showing a dominant signal intensity similar to that of fat corresponding to early mixed active phase.
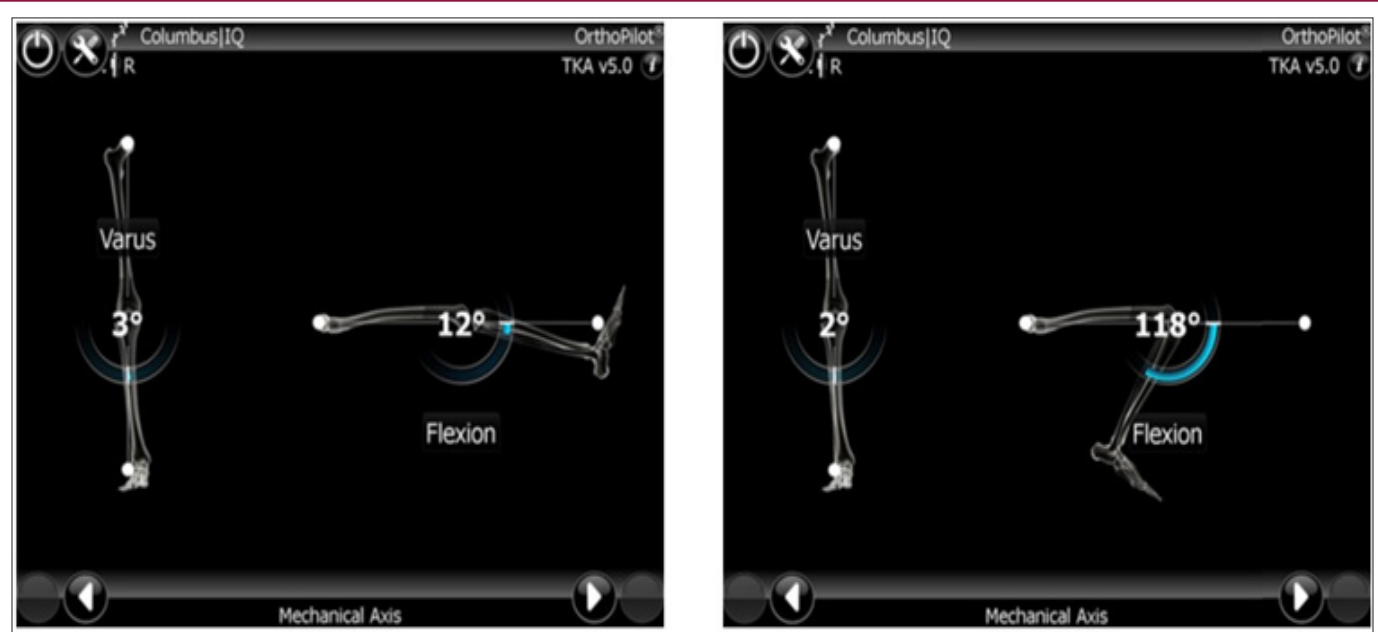

Figure 4: Computer navigation images intra-operatively showing pre-op overall varus and flexion deformities.

The pre-operative deformities recorded on navigation system were 30 varus and 120 flexion deformity (Figure 4). After appropriate soft tissue releases and bone cuts, cemented cruciate retaining implants with size 6 femur, size 3 tibia and size -10 polyethylene insert was implanted. The final alignment postoperatively was 10 valgus and 70 flexion (Figure 5). The femoral component was intentionally anteriorized and inserted in flexion to avoid any notching of anterior femoral cortex (Figure 6). There was no need for a corrective osteotomy of the femur for the extra- articular deformity. The total blood loss was $180 \mathrm{ml}$ and operative time was 62 minutes. Intra-operatively the knee was infiltrated with a cocktail mixture of $0.2 \%$ Ropivacaine- $30 \mathrm{ml}$, Ketorolac- $60 \mathrm{ml}$, Morphine $4 \mathrm{mg}, 0.5 \mathrm{ml}$ of 1:1000 Adrenaline and normal saline $30 \mathrm{ml}$. An epidural catheter was inserted in the knee and left in place for 48 hours to facilitate regular infiltration with $0.2 \%$ Ropivacaine. Apart from these measures, the patient was given an adductor canal block in the immediate post-operative period. Adductor canal block is a pure sensory block which does not affect the quadriceps function 
and the patient was made to walk on day 0 with walker support along with commencement of immediate knee mobilization. The patient was also administered 1gm Tranexamic acid IV just before the surgery. There were no drains inserted. The antibiotic prophylaxis was with $1 \mathrm{gm}$ Cefuroxime intravenous pre-operatively and two further doses post-operatively. Thromboprophylaxis was with 2.5 gm Apixaban twice a day for two weeks along with TED stockings. The patient had an uneventful recovery and was discharged on day 3 and followed up on days 14, 6weeks, 3months, 6 months and 1year. The patient returned to normal activities at 3 months and at latest follow-up of 1 year the OKS was 38 and VAS score for pain was 2 (Figure 7).
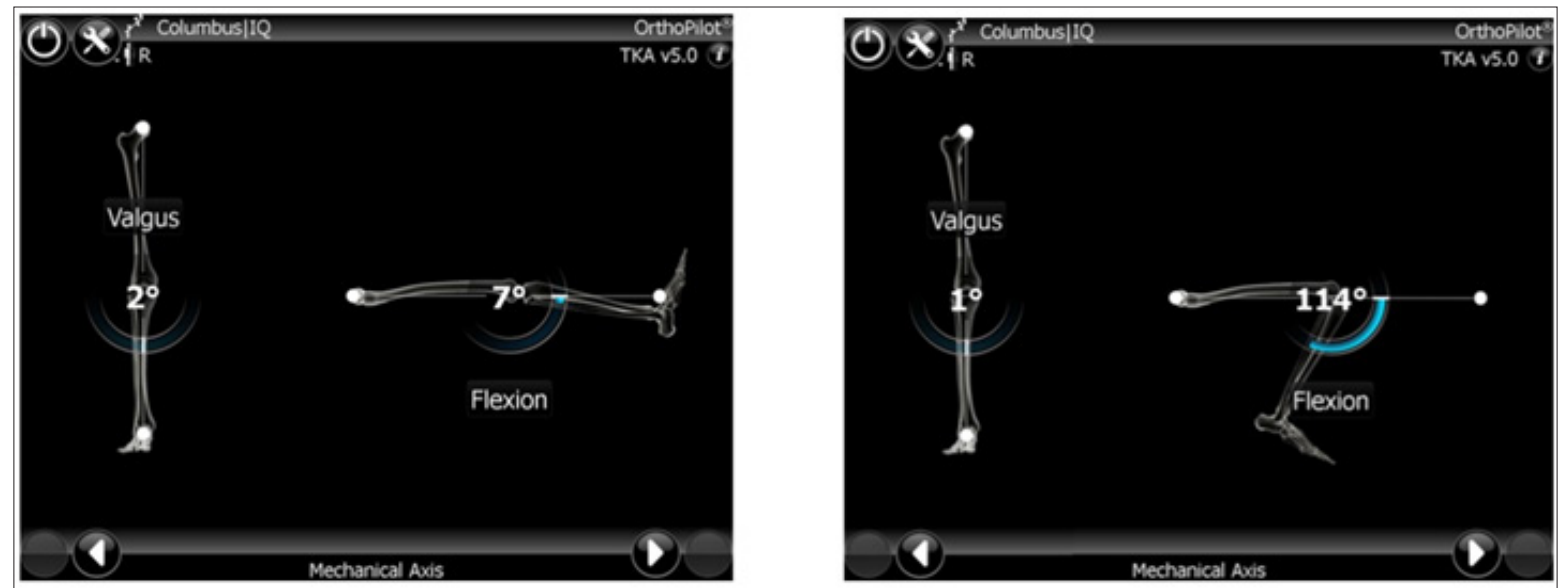

Figure 5: computer navigation images showing the final component alignment.

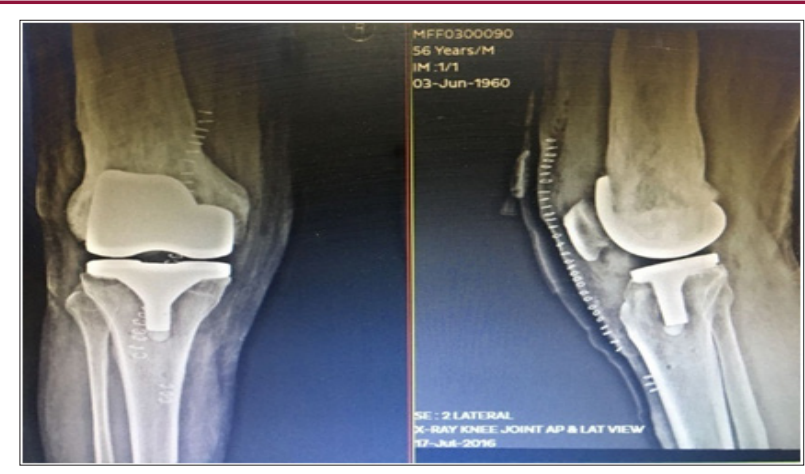

Figure 6: Immediate post-operative radiograph showing correction of deformity and restoration of mechanical alignment. Notice the femoral component in slight flexion to avoid anterior femoral notching.

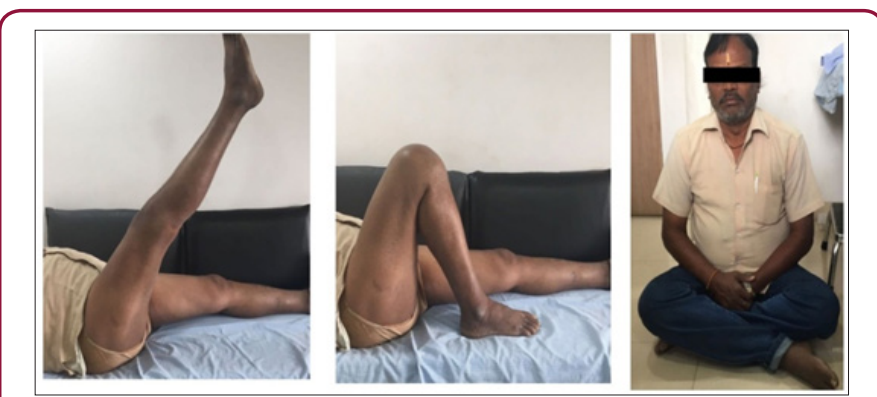

Figure 7: 1 year follow-up clinical photograph showing excellent functional.

\section{Discussion}

Paget's disease of the bone is associated with a hypervascular and hyperdynamic state and the bone is unusually hard and brittle. The differential diagnosis of Paget's is osteopetrosis, fluorosis, sclerotic secondaries. Arthroplasty surgery in a joint adjacent to a Pagetic bone poses special challenges with the amount of blood loss, increased operative time, bone hardness requiring special blades and drills to make the bone cuts and most importantly restoration of the correct mechanical alignment in view of any extra articular deformities [8,9]. These technical difficulties are well described with regards to hip arthroplasty but less literature with regards to the knee. All the reported cases in the knee are with conventional technique which reported satisfactory results but none with computer assisted surgery. Exposure of the knee can also be difficult in Paget's knee because of soft tissue contracture and hyperplastic patella [10] which we did not face in our case. When using an intramedullary guide by the conventional technique there is a possibility of femoral and tibial component size mismatch [4]. With the computer assisted surgery this problem can be overcome. In patients of Paget's with knee involvement the deformities are usually complex and multiplanar.

There could be a combination of intra and extra articular deformities and the extra-articular deformities may be in either sagittal or coronal planes along with torsional deformity. In the TKA done by conventional technique, there are reports of the knees being left outside the acceptable range of 50-100 valgus because of the difficulty in achieving a correction of the multiplanar deformities $[4,11,12]$. In the sagittal plane, because of the anterolateral bowing there is a potential risk of placing the femoral component in excessive flexion or extension by the conventional technique, in addition to the difficulty in using an intramedullary jig because of the bone hardness and risk of femoral perforation. With the help of computer assisted surgery [13], the hip, knee and ankle centres are accurately marked and potential anterior or posterior femoral notching can be avoided without affecting the flexion-extension gaps. We could also balance the knee with using a cruciate retaining prosthesis. There are previous reports of combined femoral corrective osteotomy and intramedullary nailing with simultaneous knee arthroplasty $[14,15]$ for coronal plane 
deformity more than 100 and sagittal plane deformity more than 200 . We could achieve satisfactory restoration of the alignment and balancing without the need for corrective osteotomy with the aid of computer assisted surgery.

In cases of hip arthroplasty, both cemented [16] and uncemented [17] designs have been used with good long term success. There are limited studies with regards to knee replacements; However, these have not shown any early component loosening $[4,18]$. Our limitations are short follow-up of only 1year and this being only a single case report because of the rarity of the problem in Indian population and that too monostotic involvement of the femur with knee arthritis. Computer assisted surgery is not universally available and has a steep learning curve. There are no long term studies to show the superiority of computer assisted surgery over conventional technique in terms of functional outcome. However, the senior author (KKE) was well experienced in computer assisted surgery.

\section{Conclusion}

Paget's involvement around the knee can be associated with both intra and extra-articular deformities and Total Knee Arthroplasty in these patients can be technically challenging when performed by conventional technique. Computer assisted surgery can help map the multi-planar deformity and achieve optimal overall final component alignment and ligament balancing.

\section{References}

1. Singer FR (1977) Paget's disease of bone. Plenum, New York, USA.

2. Stauffer RN, Sim FH (1976) Total hip arthroplasty in Paget's disease of the hip. J Bone Joint Surg 58(4): 476-478.

3. Mc Donald DJ, Sim FH (1987) Total hip arthroplasty in Paget's disease: a follow-up note. J Bone Joint Sur 69(5): 766-772.

4. Lee G, Sanchez-Sotelo J, Berry DJ (2005) Total Knee Arthroplasty in Patients with Paget's Disease of Bone at the Knee. J of Arthroplasty 20(6): 689-693.
5. Nugent JS, O'Brien KE, Harris M, Mohan C (2002) Paget's disease of bone in an Indian patient: genetic and environmental factors. J Clin Rheumatol 8(4): 212-216.

6. Theodorou DJ, Theodorou SJ, Kaitsubata Y (2011) Imaging of Paget disease of bone and its musculoskeletal complications: a review. Am J Roentgenol 196(6): 64-75.

7. Dawson J, Fitzpatrick R, Murray D, Carr A (1998) Questionnaire on the perceptions of patients about total knee replacement surgery. J Bone Joint Surg 80(1): 63-69.

8. Lewalen DG (1999) Hip Arthroplasty in patients with Paget's disease. Clin Orthop 369: 243-250.

9. Parvizi J, Schall DM, Lewallen DG (2002) Outcome of uncemented hip arthroplasty in patients with Paget's disease. Clin Orthop 403: 127-137.

10. Gabel GT, Rand JA, Sim FH (1991) Total knee arthroplasty for osteoarthrosis in patients who have Paget's disease of bone at the knee. J Bone Joint Surg 73(5): 739-744.

11. Wolff AM, Hungerford DS, Pepe CL (1991) The effect of extraarticular varus and valgus deformity on total knee arthroplasty. Clin Orthop Relat Res 271: 35-51.

12. Mann JW, Insall JN, Scuderi GR (1997) Total knee arthroplasty in patients with associated extra articular angular deformity. Orthop Trans 21: 59.

13. Mullaji A, Shetty GM (2009) Computer-assisted total knee arthroplasty for arthritis with extra-articular deformity. J Arthroplasty 24(8): 11641169.

14. Stephen J Incavo, Carl Kapadia, Robert Torney (2007) Use of an Intramedullary nail for Correction of Femoral Deformities Combined with Total Knee arthroplasty. J of Arthroplasty 22(1): 133-135.

15. Lonner JH, Siliski JM, Lotke PA (2000) Simultaneous femoral osteotomy and total knee arthroplasty for treatment of osteoarthritis associated with severe extra-articular deformity. J Bone Joint Surg 82(3): 342-348.

16. Ludkowski P, Wilson-Mac Donald J (1990) Total arthroplasty in Paget's disease of the hip. A clinical review and review of the literature. Clin Orthop Relat Res 255:160-167.

17. Hozack JW, Rushton AS, Carey C, Sakalkale D, Rothman RH (1999) Uncemented total hip arthroplasty in Paget's disease of the hip: a report of 5 cases with 5-year follow-up. J of Arthroplasty 14(7): 872-876.

18. Broberg MA, Cass JR (1986) Total knee arthroplasty in Paget's disease of the knee. J of Arthroplasty 1(2): 139-142.

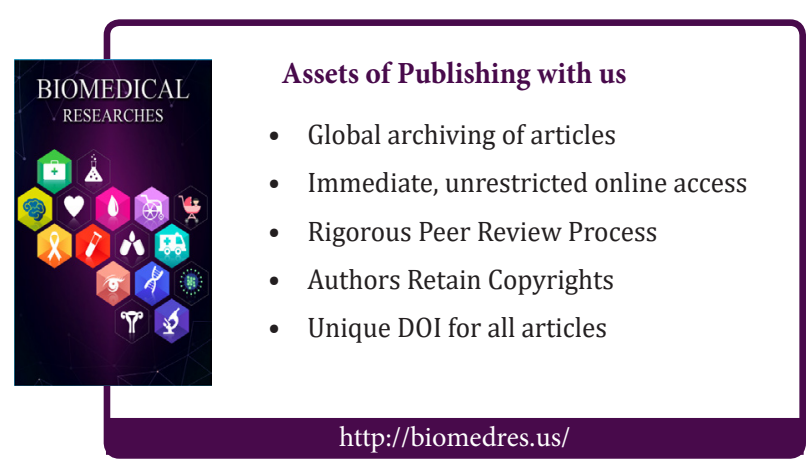

